\title{
Multiple lifestyle factors and depressed mood: a cross-sectional and longitudinal analysis of the UK Biobank $(N=84,860)$
}

Jerome Sarris ${ }^{1,2^{*}+}$, Russell Thomson ${ }^{3 \dagger}$, Fiona Hargraves ${ }^{1}$, Melissa Eaton ${ }^{1}$, Michael de Manincor ${ }^{1}$, Nicola Veronese ${ }^{4}$, Marco Solmi ${ }^{5,6}$, Brendon Stubbs ${ }^{7,8}$, Alison R. Yung ${ }^{9,10}$ and Joseph Firth ${ }^{1,10}$

\begin{abstract}
Background: There is now evolving data exploring the relationship between depression and various individual lifestyle factors such as diet, physical activity, sleep, alcohol intake, and tobacco smoking. While this data is compelling, there is a paucity of longitudinal research examining how multiple lifestyle factors relate to depressed mood, and how these relations may differ in individuals with major depressive disorder (MDD) and those without a depressive disorder, as 'healthy controls' (HC).

Methods: To this end, we assessed the relationships between 6 key lifestyle factors (measured via self-report) and depressed mood (measured via a relevant item from the Patient Health Questionnaire) in individuals with a history of or current MDD and healthy controls (HCs). Cross-sectional analyses were performed in the UK Biobank baseline sample, and longitudinal analyses were conducted in those who completed the Mental Health Follow-up.

Results: Cross-sectional analysis of 84,860 participants showed that in both MDD and HCs, physical activity, healthy diet, and optimal sleep duration were associated with less frequency of depressed mood (all $p<0.001$; ORs 0.62 to 0.94), whereas screen time and also tobacco smoking were associated with higher frequency of depressed mood (both $p<0.0001$; ORs 1.09 to 1.36). In the longitudinal analysis, the lifestyle factors which were protective of depressed mood in both MDD and HCs were optimal sleep duration (MDD OR $=1.10 ; p<0.001, \mathrm{HC} O R=1.08 ; p<0.001)$ and lower screen time (MDD OR $=0.71 ; p<0.001, \mathrm{HC} O R=0.80 ; p<0.001$ ). There was also a significant interaction between healthy diet and MDD status $(p=0.024)$, while a better-quality diet was indicated to be protective of depressed mood in $\mathrm{HCs}(\mathrm{OR}=0.92 ; p=0.045)$ but was not associated with depressed mood in the MDD sample. In a cross-sectional $(\mathrm{OR}=0.91 ; p<0.0001)$ analysis, higher frequency of alcohol consumption was surprisingly associated with reduced frequency of depressed mood in MDD, but not in HCs.

Conclusions: Our data suggest that several lifestyle factors are associated with depressed mood, and in particular, it calls into consideration habits involving increased screen time and a poor sleep and dietary pattern as being partly implicated in the germination or exacerbation of depressed mood.
\end{abstract}

Keywords: Lifestyle medicine, Mood disorders, Diet, Physical activity, Screen time, Health

\footnotetext{
* Correspondence: j.sarris@westernsydney.edu.au

${ }^{\dagger}$ Jerome Sarris and Russell Thomson contributed equally to this work.

${ }^{1}$ NICM Heath Research Institute, Western Sydney University, Westmead, NSW

2145, Australia

${ }^{2}$ Professorial Unit, The Melbourne Clinic, Department of Psychiatry, The

University of Melbourne, Melbourne, VIC, Australia

Full list of author information is available at the end of the article
}

(c) The Author(s). 2020 Open Access This article is licensed under a Creative Commons Attribution 4.0 International License, which permits use, sharing, adaptation, distribution and reproduction in any medium or format, as long as you give appropriate credit to the original author(s) and the source, provide a link to the Creative Commons licence, and indicate if changes were made. The images or other third party material in this article are included in the article's Creative Commons licence, unless indicated otherwise in a credit line to the material. If material is not included in the article's Creative Commons licence and your intended use is not permitted by statutory regulation or exceeds the permitted use, you will need to obtain permission directly from the copyright holder. To view a copy of this licence, visit http://creativecommons.org/licenses/by/4.0/. The Creative Commons Public Domain Dedication waiver (http://creativecommons.org/publicdomain/zero/1.0/) applies to the data made available in this article, unless otherwise stated in a credit line to the data. 


\section{Background}

Despite advances in pharmacological and psychological treatment options for depression, the condition is still pervasive, having a population prevalence in Western societies of approximately $7 \%$ per year $[1,2]$. Understanding potential modifiable risk factors is an essential first step to prevent the burden of depression. A recent umbrella review of non-genetic risk factors identified that six risk factors met the criteria for convincing evidence for being associated with depression, including widowhood, physical abuse during childhood, obesity, having four to five metabolic risk factors, sexual dysfunction, and job strain [3].

Recently, interest has grown in the potential relationship between lifestyle patterns and depression, in particular assessing whether people engaging in a healthy lifestyle may have a better mood compared to unhealthy counterparts. Key analyses to date tend to explore the association with physical activity [4] and also dietary quality [5], although the relationships with other modifiable lifestyle elements have been generally less explored. These lifestyle factors are all modifiable, thus providing an additional array of treatment options in concert with existing strategies. In fact, for milder forms of depression, notable treatment guidelines now recommend addressing lifestyle factors before commencing pharmacotherapy [6].

Healthy lifestyle elements are primarily considered to involve a healthy diet (higher in plant foods and lean protein, and lower in refined and ultra-processed foods); adequate (but not extreme) physical activity; adequate sleep duration and quality; avoidance or minimisation of alcohol, smoking, or substance use; and sufficient exposure to sunlight and nature [7]. Other factors which may be included within the lifestyle domain include relationship interactions (including with animals), hobbies, and time spent interfacing with technology, e.g. TV or Internet [8].

In respect to the potential neurobiological pathways through which various lifestyle factors impact mental health, data exists revealing an anti-inflammatory effect from both exercise [9] and diet [10-12]. Raised inflammation has a strong pathological link with depression. There is also data showing that smoking or poor sleep may also have pro-inflammatory effects in humans [13, 14]. Chronic low-grade inflammation has been linked with a broad range of psychiatric disorders [15]. An additional pathway modified by lifestyle factors concerns the role of the gut microbiome. The microbiome can be influenced by both physical activity [16] and diet [17], and a healthy microbiome may also potentially influence mood levels. Downstream consequences may also occur from poor health behaviours. For example, insufficient exercise, a poor diet, and insomnia can contribute factors towards the development of obesity, which in turn can raise inflammatory markers and have a negative effect on self-image and self-esteem [18]. Another simpler link can be found regarding nutrient-depleted diets not providing the vital micronutrients required for optimal neurological health [19].

In recent years, some research has assessed how a range of lifestyle factors may influence our mental wellbeing, with several cross-sectional studies, for example [20-23] revealing that other elements such as a healthy diet, adequate duration of sleep, outdoor or sunlight exposure, alcohol limitation, and smoking avoidance are beneficial behaviours which relate to general mental health and mood. This data is compelling and stands to reason due to the well-documented effects that nutrition, sleep, physical activity, sunlight and air quality, and harmful recreational substances (i.e. drugs and alcohol) have on the brain [7].

However, due to the cross-sectional design of the aforementioned studies, directionality could not yet be established. Few studies have examined the relationships of multiple lifestyle factors with future depressive symptoms. For instance, Adjibade and colleagues [24] assessed hazard ratios (estimated using Cox proportional hazards models) in addition to population attributable risks, for the mental health of a French sample of 25,837 adults, and examined the prospective relationship to five modifiable lifestyle elements (diet, smoking, alcohol consumption, weight, and physical activity). From these lifestyle factors, they created a healthy lifestyle index. After 5 years of follow-up, there were a total of 2112 cases of de novo (incident) depression, as assessed via a Centre for Epidemiological Studies Depression Scale [French version] score of $\geq 17$ and 23 for men and women (range 0-60), respectively. After accounting for confounding factors, the data revealed a significant longitudinal link between an overall healthy lifestyle and fewer emergent cases of depression.

While there is a growing body of research investigating how lifestyle factors affect the onset of depressive disorders [25], the literature has yet to address how lifestyle factors may differentially affect mood state in the general population compared to those living with major depressive disorder. Additionally, more research is needed on the potential psychological impact of increasing screen time, which has raised concerns in recent years [26]. Our rationale for conducting this study was to address these current deficits in the field and to assess multiple lifestyle measures as they are commonly interrelated. As detailed above, there is currently only limited evidence exploring the relationship between lifestyle factors and depressed mood.

To address these current deficits in the field, we conducted both a cross-sectional and a longitudinal analysis of the UK Biobank data to assess the relationship of key 
lifestyle factors (objectively measured physical activity, diet quality, adequate sleep, sedentary screen time, smoking status, and alcohol intake frequency) with frequency of depressed mood in both people with major depression and those without depression. Our a priori hypothesis was that healthy lifestyle behaviours would be associated with less depressed mood symptoms crosssectionally, while being found to be protective against depressed mood across time in a longitudinal analysis. This was undertaken as it was vital to assess lifestyle behaviours in both cohorts to contrast any differences and also to determine if a baseline depressive disorders would prospectively affect the level of depressed mood.

\section{Methods}

Generic ethical approval was granted for the UK Biobank from the NHS Research Ethics Committee (Ref. 11/NW/ 0382). The analyses presented in this study were provided separate approval by the UK Biobank research committee and were performed on data collected between 2007 and 2010 for baseline and 2016 and 2017 for longitudinal analyses. In brief, the UK Biobank is a nationwide, healthoriented, cohort study which takes place across 22 assessment centres throughout the UK [27]. The national Biobank collects data across the UK in order to investigate how various lifestyle, environmental, and genetic factors are associated with a range of health outcomes.

Potential participants were accessed via mail (sent to around 9.2 million homes in the UK). From this, a total of 502,664 participants were recruited, aged $37-73$, and were required to attend UK Biobank assessment centres for extensive data collection, which included touchscreen questionnaires, in-person interviews, and physical health examinations [27]. Further details on the aims, methods, and procedures for the UK Biobank study are available elsewhere [27].

\section{Participant sampling}

Participants used for this study were all those from the UK Biobank who could be categorised into groups of people who presented with previous or current major recurrent depression (MDD sample) and those without depressive disorders as healthy controls (HC) (i.e. no indication of present or previous affective disorders). This categorisation was based on a previous study, which assessed the prevalence of mood disorders within the UK Biobank cohort [28]. The study applied a pre-established criterion to individuals' answers to the Structured Clinical Interview for DSM-IV Axis I Disorders (SCID-1) and Patient Health Questionnaire (PHQ) [28] to categorise participants as likely having a single episode of major depression, recurrent major depression, bipolar disorder, or no indicated mood disorders. The data revealed that lifetime prevalence rates were consistent with general population-based estimates for a current unipolar depressive episode across both genders [1]. For our study, the MDD group consisted of all those falling into the categories of recurrent major depression as described above at baseline, and any individuals with a previous/current ICD10 diagnosis of recurrent major depression on UK Biobank hospital records. The HCs were those with no historical or current depressive disorder indicated from these measures at baseline. For our study, participants with neuropsychiatric conditions known to affect cognitive functioning and severe mental illnesses (such as schizophrenia other psychotic disorders and bipolar disorder) were excluded from the analyses. The supplementary material displays the excluded neurological conditions and their UK Biobank field codes.

\section{Lifestyle factor assessment}

We chose to analyse the most pertinent lifestyle-related variables we could find available on the UK Biobank database. The lifestyle factors included physical activity, diet, sleep, smoking, screen time (greater use may pertain to a more sedentary lifestyle), and alcohol frequency all of which have individually been implicated in depression [8] All of these were assessed using computerised questionnaires administered to participants at the baseline visit to UK Biobank assessment centres. The details on each of these factors and their respective UK Biobank 'Field Codes/Categories' (FCs) identifiers are given below:

\section{Physical activity}

The self-reported physical activity data available in the UK Biobank was used for this study, as only self-report physical activity was recorded immediately at baseline (while objective measures were collected later). The selfreported physical activity data were converted to metabolic equivalent of task (MET) minutes per week, using the data produced by a previous study (ref: https://bmjopen.bmj.com/content/6/3/e010038) now available within the UK Biobank (FC: 22040).

\section{Diet}

This was based on the food frequency questionnaire (FC: 100052). To determine individuals classified as having a 'healthy diet' for our analysis, the data on selfreported weekly food intake was used to determine those with (i) high intake of fruit and vegetables, (ii) high intake of fish, and (iii) low intake of processed meats and red meats. Those individuals who fulfilled at least two of those three criteria above were defined as having a 'healthy diet' (categorical variable). Further details are provided in the supplementary material for the diet categorisation used. 


\section{Sleep}

Asking 'About how many hours of sleep do you get in every $24 \mathrm{~h}$ ? (please include naps)' (FC: 1160), participants who achieved 7 to $9 \mathrm{~h}$ were classified as 'optimal sleep'. This is based off Australian Sleep Foundation Guidelines for adults (however, it is recognised that in less active older adults that 7 to $8 \mathrm{~h}$ of sleep per night may be more optimal) (https:// www.sleephealthfoundation.org.au/files/pdfs/Sleep-NeedsAcross-Lifespan.pdf).

\section{Smoking}

Using the question 'Do you smoke tobacco now?' (FC: 1239), for the purposes of this study, participants were classified as either 'non-smokers' (never or not current smokers) or 'smokers' (still current smoking).

\section{Sedentary screen time}

Sedentary screen time is calculated in hours per week, adding together the participants' answers to the questions 'In a typical DAY, how many hours do you spend watching TV?' (FC: 1070) and 'In a typical DAY, how many hours do you spend using a computer? (not including time using a computer at work)' (FC: 1080).

\section{Alcohol frequency}

Alcohol frequency is categorised as $6=$ 'daily', $5=$ 'three to four times per week', $4=$ 'once or twice per week', $3=$ 'one to three times per month', 2 = 'special occasions only', or $1=$ 'never' using participant response to the question 'About how often do you drink alcohol?' (FC: 1558). For the purposes of this study, this ordinal variable was treated as linear, so that results can be interpreted as a dose-response relationship.

\section{Depressive mood assessment}

Frequency of depressed mood were assessed over the last 2 weeks, with the following question of a computerised questionnaire administered at the baseline visit:

' Over the past two weeks, how often have you felt down, depressed or hopeless?' (FC: 2050).

And this similarly worded question, assessing the same construct of frequency of depressed mood, at follow-up (administered via an online survey):

'Over the last 2 weeks, how often have you been bothered by any of the following problems? Feeling down, depressed, or hopeless' (FC: 20510).

With participants responding as 'Prefer not to Answer', 'Not at all', 'Several days', 'More than half of days', and 'Nearly every day' in both cases.

The answers were assigned scores of missing for 'prefer not to answer' then $1,2,3$, or 4, respectively, for frequency of depressed mood, and used as an ordinal measure.

\section{Confounding variables}

Demographic information was collected from computerised questionnaires completed at UK Biobank assessment centres. Age, gender, ethnicity, social deprivation, education, and BMI were identified as the key confounding variables. Highest education/qualifications responses were dichotomized to categorise participants as either having or not having university/college degree-level qualifications. As 95\% of the sample were classified as Caucasian, ethnicity was categorised as 'Caucasian' or 'Other'. Social deprivation was assessed using the Townsend deprivation index (using participants' postcodes on joining the study). BMI measurement was acquired onsite via a research assistant who conducted the physical health assessments.

\section{Statistical analysis}

Ordinal logistic regression was used to examine the association between lifestyle factors and frequency of depressive moods in the last 2 weeks throughout this manuscript. The frequency of depressive mood variable was ordinal in nature, with four possible levels $(1=$ 'Not at all', $2=$ 'Several days', $3=$ 'More than half of days', and $4=$ 'Nearly every day'). The cross-sectional analysis used the frequency of depressive moods at the same time that the lifestyle factors were measured as the outcome variable. Lifestyle factors included physical activity (MET minutes), diet, optimal hours of sleep per night (7 to 9 h), current smoking, screen time, and alcohol frequency. The confounders for which we accounted were ethnicity, social deprivation, gender, age, and optimal BMI (18.5-25).

An ordinal logistic regression model was also fitted with the outcome variable: frequency of depressive moods at follow-up from those participants taking part in the UK Biobank study (collected from 2016 to 2017). Lifestyle factors at baseline and confounders were the predictors in this model. As the frequency of depressive moods at baseline was included as a covariate in this model, effect sizes represented change in the frequency of depressive mood from baseline to follow-up.

To assess if the lifestyle factors affect the frequency of depressive moods differently between those participants with/without a depressive disorder (MDD vs $\mathrm{HCs}$ ), separate models were fitted for these two groups. A model that included interactions between the MDD status and each of the lifestyle factors was used to test whether they affected the frequency of depressive mood differently between those with MDD and those without. This approach was important as it was theorised that those with or without pre-existing depression may have a different trajectory of mood symptoms modulated by lifestyle factors. 
For each of the models run, we reported appropriate summary statistics regarding the sample composition (sample size, averages and error surrounding measurements of interest), the model parameter estimates and associated confidence. The effect size of the lifestyle factors and confounders on the frequency of depressive mood were plotted using odds ratios and 95\% confidence intervals. Wald's test was employed for all $p$ values and confidence intervals. Statistical analyses were conducted using $\mathrm{R}$, with the help of the $\mathrm{R}$ function polr in the library, MASS. A $p$ value of $\leq 0.05$ was considered as statistically significant.

\section{Results}

\section{Sample characteristics}

The entire sample for cross-sectional analyses consisted of all 84,860 participants of the UK Biobank who (i) had provided sufficient information to establish MDD vs non-depression status, (ii) provided sufficient data on exposure and outcome measures to be included in the analyses and (iii) did not meet the exclusion criteria (as described above). The average age of the baseline sample was 55 for the MDD $(n=18,793)$ compared to 57 for the HCs $(n=66,067)$. A summary of the two groups is presented in Table 1 . The only primary difference between groups was a greater number of females (65\%) compared to males $(35 \%)$ in the sub-sample with depression. The predominance of the sample identified as being of Caucasian ethnicity (94\% and 91\%, respectively). There were also significantly more smokers in the MDD group (13\%) compared to the HCs (8\%). Finally, there was a similar BMI level for both groups.
Lifestyle factors affecting mood (cross-sectional data)

Increased physical activity, as measured by a one sd increase in MET minutes per week (MDD group: OR = $0.94,95 \%$ CI $0.91-0.96, p<0.0001$; HC group: $\mathrm{OR}=$ $0.94,95 \%$ CI $0.92-0.96, p<0.0001$ ), healthy diet (MDD group: $\mathrm{OR}=0.91,95 \% \mathrm{CI}$ 0.86-0.97, $p=0.0026$; HCs: $\mathrm{OR}=0.88,95 \%$ CI $0.84-0.92, p<0.0001)$ and optimal sleep (MDD group: $\mathrm{OR}=0.62,95 \%$ CI $0.58-0.66, p<$ 0.0001 ; HCs: $\mathrm{OR}=0.65$, 95\% CI $0.62-0.68, p<0.0001$ ) were significantly cross-sectionally associated with less depressive mood in both groups (Fig. 1, Online Table 1).

Conversely, being a current smoker (MDD group: $\mathrm{OR}=1.36,95 \%$ CI $1.25-1.48, p<0.0001$; HCs: OR = 1.32 , 95\% CI 1.22-1.42, $p<0.0001)$ and more screen time (one sd increase) (MDD group: $\mathrm{OR}=1.13,95 \% \mathrm{CI}$ $1.1-1.16, p<0.0001$; HCs: OR $=1.09,95 \%$ CI 1.07-1.12, $p<0.0001)$ were significantly associated with increased depressive mood in both groups.

Highly significant interactions were observed between a MDD status and alcohol frequency (all $p$ values $\leq$ 0.0001). Alcohol frequency had a stronger negative cross-sectional (OR $=0.91,95 \%$ CI $0.88-0.94, p<0.0001$ ) association with the frequency of depressive mood in those with MDD compared with those without depression. Cross-sectional associations with confounding variables are displayed in Online Table 1.

\section{Lifestyle factors affecting mood-longitudinal data}

In respect to the longitudinal data, the lifestyle factors screen time (MDD group: OR $=1.1,95 \%$ CI 1.05-1.15, $p=0.0001 ;$ HCs: $\mathrm{OR}=1.08,95 \%$ CI $1.04-1.12, p<$

Table 1 Number of participants and their characteristics. Midrange BMI is defined as 18.5-25. Optimal sleep is defined as 7-9 h a night. Healthy diet is defined as having 2 or more of 3 healthy diet attributes (sufficiently low meat, high fruit/vegetable, high fish). Physical activity is measured in MET minutes per week. Screen time is measured as the number of hours of non-work computer and TV time per day. Alcohol frequency is given as $1=$ 'never', 2 ='special occasions only', 3 = 'one to three times per month', $4=$ 'once or twice per week', and $5=$ 'three to four times per week'. Social deprivation measured using the Townsend deprivation index based on postcode

\begin{tabular}{llllll}
\hline & \multicolumn{2}{l}{ Baseline measures } & & & \multicolumn{2}{l}{ Baseline and follow-up measures } \\
& Depression & Non-depression & & Depression & Non-depression \\
\hline Number of participants & 18,793 & 66,067 & 7050 & 24,293 \\
Age (mean (sd)) & $55.3(8)$ & $57.1(8.1)$ & $55.1(7.7)$ & $56.8(7.8)$ \\
Women (\%) & $64.6 \%$ & $47.7 \%$ & $67.2 \%$ & $49.6 \%$ \\
Caucasian (\%) & $94.4 \%$ & $92.1 \%$ & $96.4 \%$ & $95.5 \%$ \\
Current smoker (\%) & $12.8 \%$ & $7.8 \%$ & $9.1 \%$ & $6.1 \%$ \\
Midrange BMI (\%) & $32 \%$ & $34.3 \%$ & $36.2 \%$ & $39.3 \%$ \\
Optimal sleep (\%) & $70.5 \%$ & $76.3 \%$ & $74.1 \%$ & $38.8 \%$ \\
Healthy diet (\%) & $38.3 \%$ & $37 \%$ & $39 \%$ & $37.4 \%$ \\
Physical activity (mean (sd)) & $2663(2743)$ & $2765(2712)$ & $2493(2488)$ & $2588(2458)$ \\
Screen time (mean (sd)) & $3.9(2.4)$ & $3.8(2.2)$ & $3.8(2.3)$ & $3.7(2.1)$ \\
Alcohol frequency (mean (sd)) & $4(1.6)$ & $4.2(1.5)$ & $4.1(1.5)$ & $-1.2(2.8)$ & $4.4(1.4)$ \\
Social deprivation (mean (sd)) & $-1(3)$ & $-1.4(2.8)$ & $-1.7(2.6)$ \\
\hline
\end{tabular}




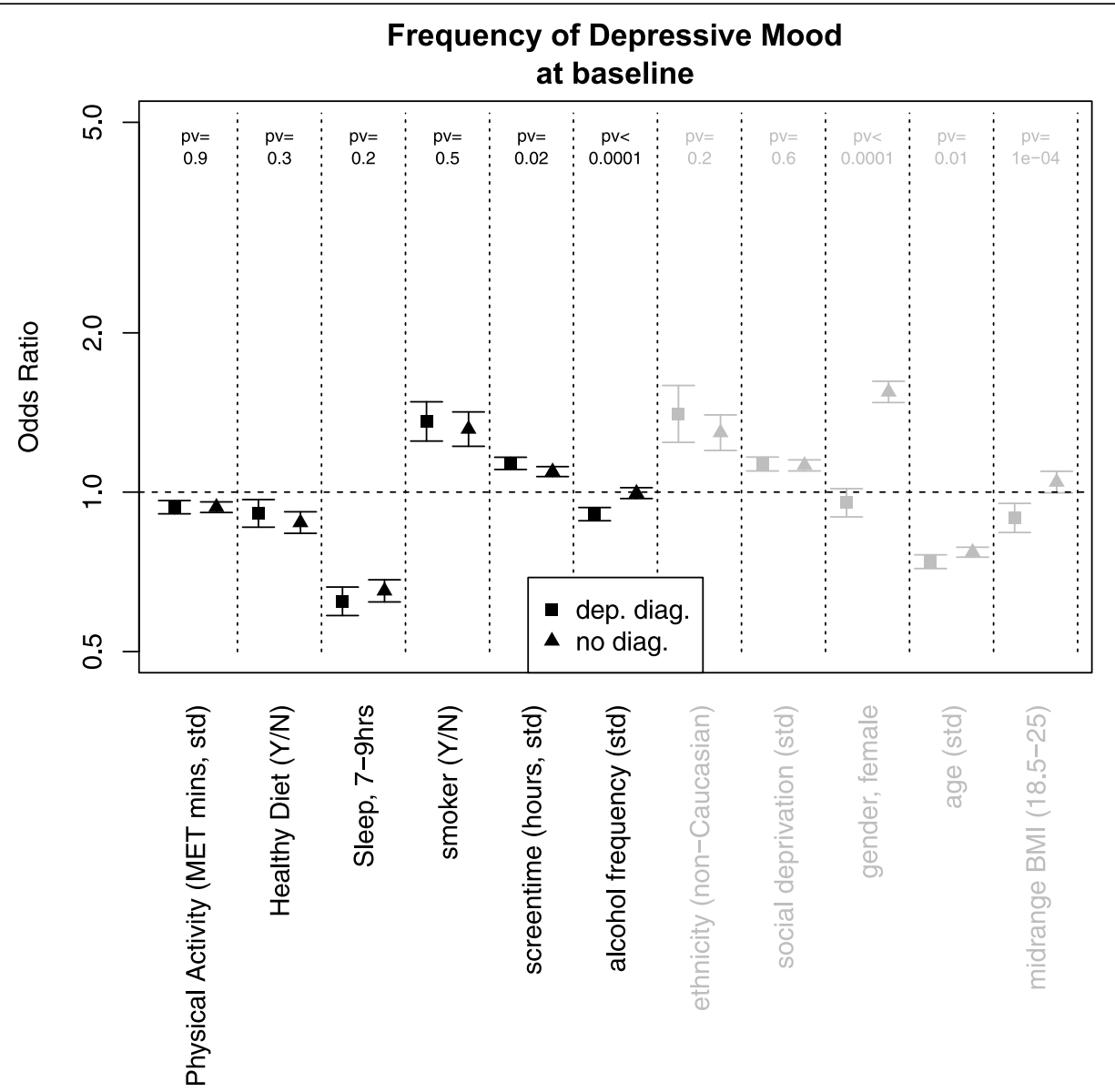

Fig. 1 The odds ratios (and 95\% confidence intervals) for an increased frequency of depressive mood at baseline, for a number of lifestyle factors and confounders (in grey) measured at baseline. Results are presented both for the samples with major depressive disorder (squares) and those without a depressive disorder (triangles). Odds ratios above 1 indicate that an increase in the given lifestyle factor is associated with more depressive moods at a similar time point. For the categorical variables (sleep, 7-9 h; smoker; ethnicity (non-Caucasian); gender (female); and midrange BMI), the reference group is all other participants. std = standardised variables. $p v=p$-values for interaction

0.0001 ) and optimal sleep (MDD group: $\mathrm{OR}=0.71,95 \%$ CI $0.64-0.8, p<0.0001$; HCs: OR $=0.80,95 \%$ CI $0.74-$ $0.87, p<0.0001)$ were prospectively associated with depressed mood at follow-up for both the MDD group and HCs. These models adjusted for baseline depressed mood, indicating that more screen time and non-optimal sleep were associated with an increase in the frequency of depressed mood from baseline to follow-up (Fig. 2).

There was also a significant interaction between healthy diet and MDD status $(p=0.024)$. A healthy diet at baseline was significantly associated with a decrease in the frequency of depressed moods at follow-up in the HCs $(\mathrm{OR}=0.92,95 \%$ CI $0.85-0.998, p=0.045)$ but not in the MDD group $(\mathrm{OR}=$ 1.07, 95\% CI $0.97-1.19, p=0.20$ ). Longitudinal associations with confounding factors are displayed in Online Table 2.

\section{Discussion}

The data from one of the largest analyses of lifestyle data and mental health showed, as expected, that a range of elements were associated with increased depressive moods in the cross-sectional analyses. Results provided a strong indication that optimal sleep with less screen time may protect against depressive symptoms over time, in both those with depressive disorders and those without. Having a healthy diet was associated with reduced frequency of depressed mood at follow-up in HCs, but not those with pre-existing depressive disorders. Other factors, such as being a current smoker and physical activity levels, were not prospectively associated with depressed mood at follow-up. A likely reason the physical activity (PA) variable did not align with our hypothesis regarding the potential protective effect of lifestyle behaviours on depressed mood concerns the discrepancy between self-reported and 'actual' PA [29]. Indeed, previous research in the UK Biobank has found objectively measured physical activity is protective of depression onset, while self-reported levels of physical activity are not [30], and the same may apply for depressive moods. 


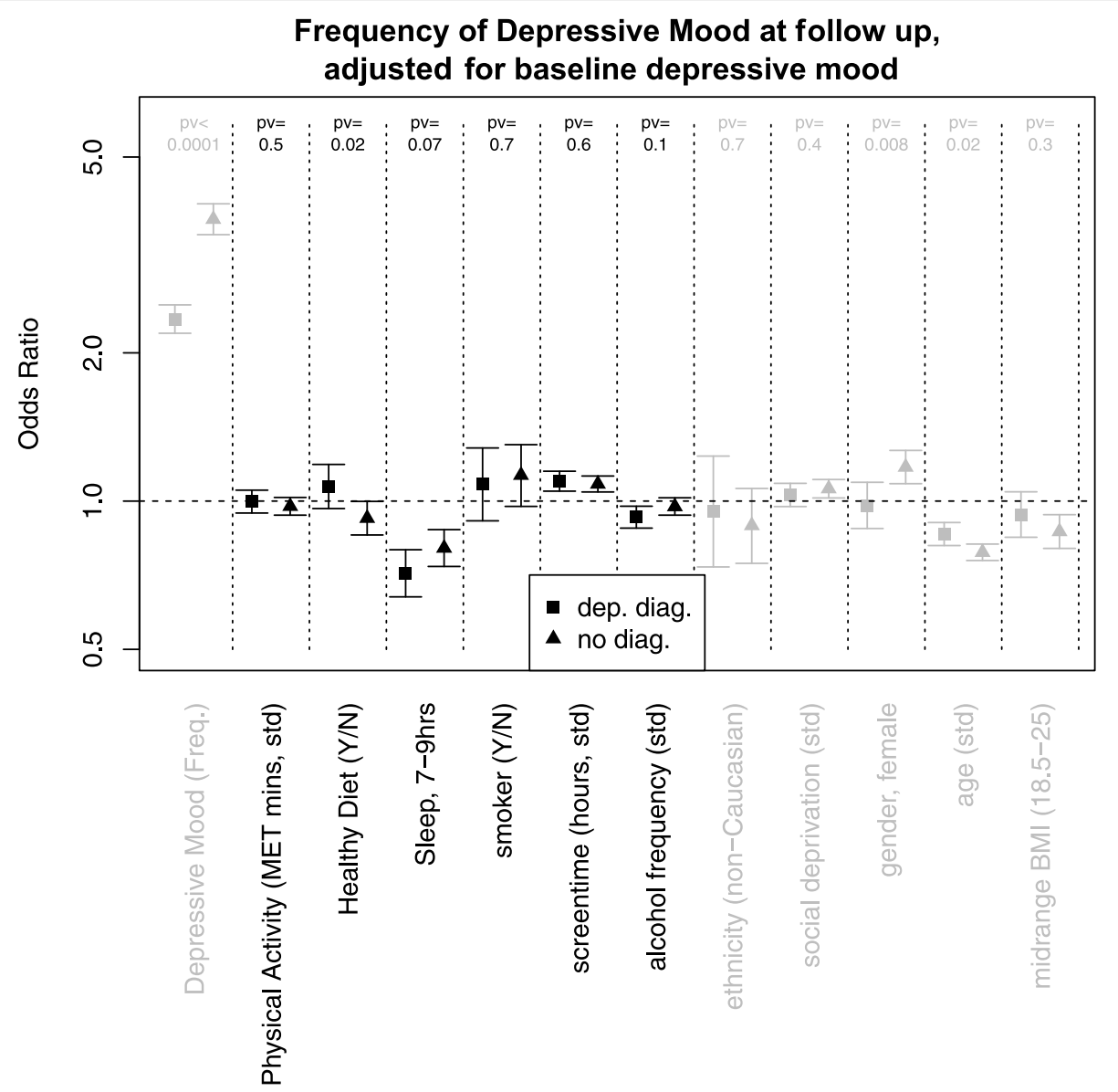

Fig. 2 The odds ratios (and 95\% confidence intervals) for an increased frequency of depressive mood at baseline and at follow-up, for a number of lifestyle factors and confounders (in grey) measured at baseline. The variable labelled 'Depressive Mood (Freq.)' is measured at baseline. Results are presented both for the samples with major depressive disorder (squares) and those without a depressive disorder (triangles). Odds ratios above 1 indicate that an increase in the given lifestyle factor is associated with an increase in depressive moods from baseline to follow-up. For the categorical variables (sleep, 7-9 h; smoker; ethnicity (non-Caucasian); gender (female); and midrange BMI), the reference group is all other participants. $s t d=$ standardised variables. $p v=$-values for interaction

However, due to the objective physical activity data within the UK Biobank cohort being collected several years after baseline assessments, only the self-report measures were suitable for our analyses. Another curious finding concerned the observation that a higher frequency of alcohol consumption was associated with lower frequency of depressive moods among those with MDD. While the damage of heavy alcohol use has been well-established, it is possible that people with depression may use alcohol as a form of self-medication, which for some may reduce the subjective experience of depressed mood being replaced by effects of alcohol. Overall, the effect size was small and neglectable compared with other detrimental effects of alcohol.

The cross-sectional findings are in concert with the majority of research, with the longitudinal data in part aligning with Adjibade and colleagues [24] who found a prospective relationship to five lifestyle/health elements (smoking, regular physical activity, diet quality, alcohol consumption and BMI). Their formulated healthy lifestyle index revealed a significant prospective link between overall healthy lifestyle and fewer de novo cases of depression. We do acknowledge that the use of a form of healthy lifestyle index may however be a helpful approach, as it is important to capture if possible a potential cumulative effect of healthy lifestyle behaviours on reducing depressive symptoms. To date, however, such indexes have not been validated, and further work is needed before adopting this as a standard approach. As recent work has found that many lifestyle and social factors which appear to contribute towards depression onset in prospective analyses may not hold causal relations [25], further research is also required to determine the underpinning causality of lifestyle factors with regards to depressive moods in both those with MDD and the general population. 
Our results may inform public health policy by further highlighting the important relationship between people being encouraged and supported to engage in a range of health-promoting activities, in particular maintaining optimal sleep and lessening screen time, may reduce the frequency of depressive moods in those with and without depressive disorders. It is interesting to note that those without depression who had a healthier diet at baseline had a significantly lower risk of depressive symptoms at follow-up, while this effect was not evident in those with MDD at baseline. One explanation for this could potentially be due to those with depression having a pre-existent poorer dietary pattern, which was maintained over time. In respect to the relationship between higher screen time with increased frequency of depressed mood, it has yet to be established if these relations are due to the sedentary nature of screen time activities or due to the potential 'direct' psychological impact of content absorbed from TV time/computer time itself. In either case, this may also be coupled as an exacerbatory factor with the established link between poor sleep and increased risk of depression [31], as high levels of screen time may displace sleep time or otherwise negatively impact on sleep quality itself.

In respect to interventional approaches, there is solid data in terms of prescriptive exercise to improve mood, while there is less so for other modifiable lifestyle elements. The evidence underpinning dietary programmes as reducing depressive symptoms is also evolving [32], and a number of recent RCTs have further found that Mediterranean diet interventions have moderately large effects on symptoms of depression in those with clinical depressive disorders [33-35]. Other meta-analyses of non-pharmacological sleep enhancement programmes have also found evidence for reductions in depressive symptoms [36]. Reduction of harmful behaviours such as smoking or excessive alcohol misuse has also been shown to improve mood [37]. What is less known, however, is the impact of actively reducing screen time, interaction with excessive social media and multi-tasking interface with technology [38, 39].

From a clinical perspective, lifestyle programmes need to be supportive, personalised and ideally consist of a multi-component approach, especially to address physical comorbidities which often co-exist with depression. While the findings in our paper do not support the relationship between all lifestyle factors modifying depressed mood over time, the combination of the cross-sectional and longitudinal data, considered alongside the findings of other recent studies, does support the use of multipronged lifestyle intervention as being an effective approach (as embryonic RCT data has revealed [40]). An example of a multi-pronged lifestyle intervention is shown in the recently published NEWTx pilot study, which examined the intervention of nutrition, exercise and wellness via general education modules in 38 people with bipolar disorder (19 vs 19; active programme vs waitlist; assessors were blinded) [41]. While the treatment group reported a range of beneficial effects on their wellbeing and symptoms, only improvements in general functioning were significantly greater in the NEWTx group than in the control group. There were no group differences in weight loss or mood symptoms over the study duration (although the treatment group did have a trend for a reduction of no over time). The authors commented that a manualised generalised approach does not appear to be sufficient, and a more personalised approach according to their needs (e.g. physical capability, medications, diagnoses) is needed to achieve a greater effect. This is in keeping with the recommendations of the recent Lancet Psychiatry Commission regarding the delivery of multi-component lifestyle interventions for people with mental illness [42].

While our paper reflects several strengths, including both the cross-sectional and longitudinal analysis of the largest cohort with lifestyle and mood data to date, limitations are recognised for this analysis. Principally, it is acknowledged that most of the lifestyle data collected via the UK Biobank were based on self-report measures, which have been shown to produce inaccurate representations of lifestyle risk factors [29] It is also understood that the UK Biobank is not a perfect socioeconomic representation of UK residents and that selection bias into the study occurred (due in part to a potential overrepresentation of an older more educated Caucasian population). Analyses were conducted using listwise deletion, and as such, the results represent the sample where all data was available. A further limitation is that depressed mood was not assessed via a specialised clinician-rated depression scale (such as the Hamilton Depression Rating Scale), and instead based on a singleitem measure of the recent frequency of depressed mood. The strengths however include the large sample, separation of participants into MDD and $\mathrm{HC}$ at baseline, and prospective nature of the analyses.

\section{Conclusions}

We conclude from our analysis of the UK Biobank lifestyle and mood data that over all, a range of lifestyle factors are related to depression and a range of lifestyle factors were found to potentially provide a significant protective factor for the frequency of depressive mood at longitudinal follow-up. Future research should look at more complex modelling to assess the potential of various lifestyle elements to influence mood. The design of interventions should also consider more integrated approaches. This is best enacted via supported individualised, tailored formats which are adaptable to participants' needs and capabilities. 


\section{Supplementary information}

Supplementary information accompanies this paper at https://doi.org/10. 1186/s12916-020-01813-5.

Additional file 1: Table S1. Results of the cross-sectional analysis on the association between frequency of depressive moods and lifestyle factors and confounders. Table S2. Results of the longitudinal analysis on the association between frequency of depressive moods at follow-up and lifestyle factors and confounders. Table S3. Excluded neuropsychiatric disorders from UK Biobank records.

\section{Acknowledgements}

We would firstly like to thank the participants and staff of the UK Biobank for making this research possible. JS is supported by an NHMRC Clinical Research Fellowship APP1125000. JF is supported by a University of Manchester Presidential Fellowship (P123958) and a UK Research and Innovation Future Leaders Fellowship (MR/T021780/1) and has received support from a NICM-Blackmores Institute Fellowship. This research has been conducted using the UK Biobank Resource under Application Number 22125 BS is supported by a Clinical Lectureship (ICA-CL-2017-03-001) jointly funded by Health Education England (HEE) and the National Institute for Health Research (NIHR); he received funding from the NIHR Biomedical Research Centre at South London and the Maudsley NHS Foundation Trust. BS is also supported by the Maudsley Charity, King's College London and the NIHR South London Collaboration for Leadership in Applied Health Research and Care (CLAHRC). This paper presents independent research. The views expressed in this publication are those of the authors and not necessarily those of the acknowledged institutions. ARY is supported by an NHMRC Principal Research Fellowship.

\section{Authors' contributions}

$J S, J F$, and RT conceived the data analysis plan and wrote the initial draft of the manuscript. RT analysed the data. All others contributed to developing the intellectual focus of the publication and assisted in the editing of the manuscript. All authors read and approved the final manuscript.

\section{Funding}

No funding specific to this publication has been provided.

\section{Availability of data and materials}

The data used in the analyses contained within the publication are available via the UK Biobank pending their consent for access.

\section{Ethics approval and consent to participate}

Covered under the over-arching UK Biobank ethics.

\section{Consent for publication}

All authors provide consent for publication.

\section{Competing interests}

No relevant conflicts of interest noted.

\section{Author details}

${ }^{1}$ NICM Heath Research Institute, Western Sydney University, Westmead, NSW 2145, Australia. ${ }^{2}$ Professorial Unit, The Melbourne Clinic, Department of Psychiatry, The University of Melbourne, Melbourne, VIC, Australia. ${ }^{3}$ Centre for Research in Mathematics and Data Science, Western Sydney University, Parramatta, NSW, Australia. ${ }^{4}$ National Research Council, Neuroscience Institute, Aging Branch, Padua, Italy. ${ }^{5}$ Neurosciences Department, University of Padua, Padua, Italy. ${ }^{6}$ Padua Neuroscience Center, University of Padua, Padua, Italy. ${ }^{7}$ Physiotherapy Department, South London and Maudsley NHS Foundation Trust, Denmark Hills, London SE5 8AZ, UK. ${ }^{8}$ Department of Psychological Medicine, Institute of Psychiatry, Psychology and Neuroscience, King's College London, De Crespigny Park Box, London SE5 8AF, UK. ${ }^{9}$ Orygen, Department of Psychiatry, Melbourne University, Melbourne, VIC, Australia. ${ }^{10}$ Division of Psychology and Mental Health, University of Manchester, Manchester, UK.
Received: 18 May 2020 Accepted: 11 October 2020

Published online: 12 November 2020

\section{References}

1. Wittchen HU, Jacobi F, Rehm J, Gustavsson A, Svensson M, Jonsson B, Olesen J, Allgulander C, Alonso J, Faravelli C, et al. The size and burden of mental disorders and other disorders of the brain in Europe 2010. Eur Neuropsychopharmacol. 2011;21(9):655-79.

2. Murphy JA, Sarris J, Byrne GJ. A review of the conceptualisation and risk factors associated with treatment-resistant depression. Depress Res Treat. 2017;2017:4176825.

3. Kohler CA, Evangelou E, Stubbs B, Solmi M, Veronese N, Belbasis L, Bortolato B, Melo MCA, Coelho CA, Fernandes BS, et al. Mapping risk factors for depression across the lifespan: an umbrella review of evidence from metaanalyses and Mendelian randomization studies. J Psychiatr Res. 2018;103: 189-207.

4. Schuch FB, Vancampfort D, Firth J, Rosenbaum S, Ward PB, Silva ES, Hallgren M, Ponce De Leon A, Dunn AL, Deslandes AC et al: Physical activity and incident depression: a meta-analysis of prospective cohort studies. Am J Psychiatry 2018, 175(7):631-648.

5. Quirk SE, Williams L, O'Neil A, Pasco JA, Jacka FN, Housden S, Berk M, Brennan SL. The association between diet quality, dietary patterns and depression in adults: a systematic review. BMC psychiatry. 2013;13:175.

6. Malhi GS, Outhred T, Hamilton A, Boyce PM, Bryant R, Fitzgerald PB, Lyndon B, Mulder R, Murray G, Porter RJ, et al. Royal Australian and New Zealand College of Psychiatrists clinical practice guidelines for mood disorders: major depression summary. Med J Aust. 2018;208(4):175-80.

7. Berk M, Sarris J, Coulson CE, Jacka FN. Lifestyle management of unipolar depression. Acta Psychiatr Scand Suppl. 2013;443:38-54.

8. Sarris J, O'Neil A, Cousan C, Berk M. Lifestyle medicine for depression. BMC Psychiatry. 2014;10(14):107.

9. Morgan JA, Olagunju AT, Corrigan F, Baune BT. Does ceasing exercise induce depressive symptoms? A systematic review of experimental trials including immunological and neurogenic markers. J Affect Disord. 2018;234: 180-92.

10. Salari-Moghaddam A, Saneei P, Larijani B, Esmaillzadeh A. Glycemic index, glycemic load, and depression: a systematic review and meta-analysis. Eur J Clin Nutr. 2019;73(3):356-65.

11. O'Keefe JH, Gheewala NM, O'Keefe JO. Dietary strategies for improving postprandial glucose, lipids, inflammation, and cardiovascular health. J Am Coll Cardiol. 2008:51(3):249-55.

12. Kiecolt-Glaser JK, Fagundes CP, Andridge R, Peng J, Malarkey WB, Habash D, Belury MA. Depression, daily stressors and inflammatory responses to highfat meals: when stress overrides healthier food choices. Mol Psychiatry. 2017;22(3):476-82.

13. Krysta K, Krzystanek M, Bratek A, Krupka-Matuszczyk I. Sleep and inflammatory markers in different psychiatric disorders. J Neural Transm. 2017:124(1):179-86.

14. Hamer M, Molloy GJ, de Oliveira C, Demakakos P. Persistent depressive symptomatology and inflammation: to what extent do health behaviours and weight control mediate this relationship? Brain Behav Immun. 2009; 23(4):413-8.

15. Yuan N, Chen Y, Xia Y, Dai J, Liu C. Inflammation-related biomarkers in major psychiatric disorders: a cross-disorder assessment of reproducibility and specificity in 43 meta-analyses. Transl Psychiatry. 2019;9(1):1-13.

16. Mailing $L$, Allen JM, Buford TW, Fields $C J$, Woods JA. Exercise and the gut microbiome: a review of the evidence, potential mechanisms, and implications for human health. Exerc Sport Sci Rev. 2019;47(2):75-85.

17. Ghosh T, Rampelli S, Jeffery I: Mediterranean diet intervention alters the gut microbiome in older people reducing frailty and improving health status: the NU-AGE 1-year dietary intervention across five European countries. Gut 2020:https://doi.org/10.1136/gutjnl-2019-319654

18. Ambrosio G, Kaufmann FN, Manosso L, Platt N, Ghisleni G, Rodrigues ALS, Rieger DK, Kaster MP. Depression and peripheral inflammatory profile of patients with obesity. Psychoneuroendocrinology. 2018;91:132-41.

19. Sarris J, Logan AC, Akbaraly TN, Amminger GP, Balanza-Martinez V, Freeman MP, Hibbeln J, Matsuoka Y, Mischoulon D, Mizoue T, et al. Nutritional medicine as mainstream in psychiatry. Lancet Psychiatry. 2015;2(3):271-4.

20. Xu Y, Qi J, Yang Y, Wen $X$. The contribution of lifestyle factors to depressive symptoms: a cross-sectional study in Chinese college students. Psychiatry Res. 2016;245:243-9. 
21. Atzendorf J, Apfelbacher C: Patterns of multiple lifestyle risk factors and their link to mental health in the German adult population: a cross-sectional study. 2018, 8(12):e022184.

22. Loprinzi PD, Mahoney S. Concurrent occurrence of multiple positive lifestyle behaviors and depression among adults in the United States. J Affect Disord. 2014;165:126-30.

23. Gomes de Matos E, Kraus L, Piontek D, Buttery AK, Mensink GB, Busch MA: Healthy behaviours and mental health: findings from the German Health Update (GEDA). BMJ Open 2015, 25(2):219-225.

24. Adjibade M, Lemogne C, Julia C, Hercberg S, Galan P, Assmann KE, KesseGuyot E. Prospective association between combined healthy lifestyles and risk of depressive symptoms in the French NutriNet-Sante cohort. J Affect Disord. 2018;238:554-62

25. Choi KW, Stein MB, Nishimi KM, Ge T, Coleman JRI, Chen CY, Ratanatharathorn A, Zheutlin AB, Dunn EC, andMe Research T et al: An exposure-wide and Mendelian randomization approach to identifying modifiable factors for the prevention of depression. Am J Psychiatry 2020: appiajp202019111158.

26. Firth J, Torous J, Stubbs B, Firth JA, Steiner GZ, Smith L, Alvarez-Jimenez M, Gleeson J, Vancampfort D, Armitage CJ, et al. The "online brain": how the Internet may be changing our cognition. World Psychiatry. 2019;18(2):119-29.

27. U.K.Biobank: The UK Biobank: rationale, design and development of a largescale prospective resource. In.: UK Biobank Coordinating Centre, Stockport, UK; 2007.

28. Smith DJ, Nicholl BI, Cullen B, Martin D, Ul-Haq Z, Evans J, Gill JM, Roberts B, Gallacher J, Mackay D. Prevalence and characteristics of probable major depression and bipolar disorder within UK biobank: cross-sectional study of 172,751 participants. PLoS One. 2013;8(11):e75362.

29. Firth J, Stubbs B, Vancampfort D, Schuch FB, Rosenbaum S, Ward PB, Firth JA, Sarris J, Yung AR. The validity and value of self-reported physical activity and accelerometry in people with schizophrenia: a population-scale study of the UK Biobank. Schizophr Bull. 2018:44(6):1293-300.

30. Choi KW, Chen CY, Stein MB, Klimentidis YC, Wang MJ, Koenen KC, Smoller JW, Major Depressive Disorder Working Group of the Psychiatric Genomics C. Assessment of bidirectional relationships between physical activity and depression among adults: a 2-sample Mendelian randomization study. JAMA Psychiatry. 2019;76(4):399-408.

31. Baglioni C, Battagliese G, Feige B, Spiegelhalder K, Nissen C, Voderholzer U, Lombardo C, Riemann D. Insomnia as a predictor of depression: a metaanalytic evaluation of longitudinal epidemiological studies. J Affect Disord. 2011;135(1-3):10-9.

32. Firth J, Marx W, Dash S, Carney R, Teasdale S, Solmi M, Stubbs B, Schuch F, Carvalho A, Jacka $F$ et al: The effects of dietary improvement on symptoms of depression and anxiety: a meta-analysis of randomised controlled trials. Psychosom Med 2019:doi: https://doi.org/10.1097/PSY.0000000000000673.

33. Parletta N, Zarnowiecki D, Cho J, Wilson A, Bogomolova S, Villani A, Itsiopoulos C, Niyonsenga T, Blunden S, Meyer B, et al. A Mediterraneanstyle dietary intervention supplemented with fish oil improves diet quality and mental health in people with depression: a randomized controlled trial (HELFIMED). Nutr Neurosci. 2019;22(7):474-87.

34. Jacka FN, O'Neil A, Opie R, Itsiopoulos C, Cotton S, Mohebbi M, Castle D, Dash S, Mihalopoulos C, Chatterton ML, et al. A randomised controlled trial of dietary improvement for adults with major depression (the 'SMILES' trial). BMC Med. 2017;15(1):23.

35. Francis HM, Stevenson RJ, Chambers JR, Gupta D, Newey B, Lim CK. A brief diet intervention can reduce symptoms of depression in young adults - a randomised controlled trial. PLOS One. 2019;14(10):e0222768.

36. Gee B, Orchard F, Clarke E, Joy A, Clarke T, Reynolds S. The effect of nonpharmacological sleep interventions on depression symptoms: a metaanalysis of randomised controlled trials. Sleep Med Rev. 2019;43:118-28.

37. Taylor G, McNeill A, Girling A, Farley A, Lindson-Hawley N, Aveyard P: Change in mental health after smoking cessation: systematic review and meta-analysis. BMJ (Online) 2014, 348 (no pagination)(g1151).

38. Liu M, Wu L, Yao S. Dose-response association of screen time-based sedentary behaviour in children and adolescents and depression: a metaanalysis of observational studies. Br J Sports Med. 2016;50(20):1252-8.

39. Firth J, Torous J, Stubbs B, Firth J, Steiner G, Smith L, Sarris J: The "online brain": how is the internet changing cognition? World Psychiatry 2019:in press.

40. Garcia-Toro M, Ibarra O, Gili M, Serrano MJ, Olivan B, Vicens E, Roca M. Four hygienic-dietary recommendations as add-on treatment in depression: a randomized-controlled trial. J Affect Disord. 2012;140(2):200-3.
41. Sylvia LG, Pegg SL, Dufour SC, Janos JA, Bernstein EE, Chang WC, Hall NE, Ellard KK, Nierenberg AA, Deckersbach T. Pilot study of a lifestyle intervention for bipolar disorder: nutrition exercise wellness treatment (NEW Tx). J Affect Disord. 2019;250:278-83.

42. Firth J, Siddiqi N, Koyanagi A, Siskind D, Rosenbaum S, Galletly C, Allan S, Caneo C, Carney R, Carvalho A et al: The Lancet Psychiatry Commission: a blueprint for protecting physical health in people with mental illness. The Lancet Psychiatry 2019, in press: doi: https://doi.org/10.1016/S22150366(1019)30132-30134

\section{Publisher's Note}

Springer Nature remains neutral with regard to jurisdictional claims in published maps and institutional affiliations.
Ready to submit your research? Choose BMC and benefit from:

- fast, convenient online submission

- thorough peer review by experienced researchers in your field

- rapid publication on acceptance

- support for research data, including large and complex data types

- gold Open Access which fosters wider collaboration and increased citations

- maximum visibility for your research: over $100 \mathrm{M}$ website views per year

At $\mathrm{BMC}$, research is always in progress.

Learn more biomedcentral.com/submissions 